\title{
Estimation of the mass transport parameters of aquifers according to data from field tests with a pulse or a continuous source and an arbitrary location of the observation well
}

Nikolay Stoyanov ${ }^{l}$

${ }^{1}$ University of Mining and Geology "St. Ivan Rilski”, Sofia;

e-mail: nts@mgu.bg

\section{Определяне миграционните параметри на} водоносния хоризонт по данни от полеви опити с импулсен или непрекъснат източник при произволно разположен наблюдателен сондаж

\section{Николай Стоянов}

Stoyanov, N. 2021. Estimation of the mass transport parameters of aquifers according to data from field tests with a pulse or a continuous source and an arbitrary location of the observation well. Engineering Geology and Hydrogeology, 35, 71-82.

\begin{abstract}
An identification method for determining the aquifer's mass transport parameters is proposed, based on data from field tracer tests with a pulse or a continuous source and an arbitrary position of the observation well in respect to the tracer entry point. The method is also applicable in the presence of a representative set of data on changes in the concentration of pollutants at different points in the aquifer around a short-term (instantaneous) or a continuous surface or underground source. The identification procedure is based on the automated comparison of the observations data with a series of theoretical curves by varying the required parameters in order to achieve maximum compliance. The tracer transport is represented by analytical solutions of the partial differential equation for mass transfer in a homogeneous and isotropic two-dimensional porous media. The developed computer programs include numerical optimization using the Levenberg-Marquardt algorithm. Results from tests performed in order to assess reliability and errors of detection and identification are presented. Using the programs, the mass transport parameters: active porosity $n_{0}$, effective (sorption) porosity $n_{S}$, longitudinal dispersivity $\alpha_{L}$, transverse dispersivity $\alpha_{T}$ and rate constant $\gamma$ can be determined.
\end{abstract}

Keywords: groundwater pollution; mass transport parameters; field tracer tests, methods for identification of hydrogeological parameters.

Резюме. Предложен е идентификационен метод за определяне миграционните параметри на водоносния хоризонт по данни от полеви трасерни опити с импулсен или непрекъснат източник при произволно положение на наблюдателния сондаж спрямо точката на постьпване на индикатора. Методът е приложим и при наличие на представителна редица от данни за 
промените в концентрацията на замърсителите в различни точки на водоносния хоризонт около краткотраен (мигновен) или непрекъснат повърхностен или подземен източник на замърсяване. Идентификационната процедура се базира на автоматизирано сравняване на данните от наблюденията с поредица от теоретични криви посредством вариране на търсените параметри до достигане на максимално съответствие. Движението на индикатора се представя посредством аналитични решения на частното диференциално уравнение за пренос на вещество в хомогенна и изотропна двумерна пореста среда. Съставените компютърни програми включват оптимизационна схема, използваща алгоритъма на Levenberg-Marquardt. Представени са резултати от проведените тестове за оценка на идентификационната грешка и за шумоустойчивостта на решението. Посредством програмите могат да бъдат определени миграционните параметри активна порестост $n_{0}$, сорбционната порестост $n_{S}$, надлъжна дисперсивност $\alpha_{L}$, напречна дисперсивност $\alpha_{T}$ и коефициент на необратимо елиминиране $\gamma$.

Ключови думи: замърсяване на подземните води, миграционни параметри, полеви трасерни тестове, методи за идентификация на хидрогеоложките параметри.

\section{Въведение}

Хидрогеоложките изчисления за оценка и прогнозиране замърсяването на водоносните хоризонти изискват добро познаване на миграционните параметри на водовместващата среда спрямо различни групи и видове замърсители. Тези параметри характеризират количествено процесите съпътстващи конвективния пренос на вещество и водят до забавяне на разпространението на замърсителите в подповърхностното пространство. Участват като константи в основното диференциално уравнение на миграцията. По тази причина тяхното определяне е много важен етап от хидрогеоложките проучвания при решаването на практически задачи.

Основните миграционни параметри активна порестост $n_{0}$, сорбционна порестост $n_{s}$, коефициент на разпределение $K_{d}$, дисперсивност $\alpha$ и коефициент на елиминиране $\gamma$ обикновено се определят посредством лабораторни или полеви трасерни опити. Прилагат се различни опитни схеми с непрекъснато или импулсно (пакетно) подаване на индикатора в пусковия пункт - опити в колони, опити в естествен поток, водочерпателни опити, нагнетателни опити, дублетни опити и др. Опитите най-често се изпълняват в условията на едномерен пренос на вещество, като пусковият и наблюдателният сондаж се разполагат по протежението на една токова линия. Това позволява използване на опростени аналитични решения за описване на протичащия в хода на опита миграционен процес. Доста по-рядко опитите се провеждат в условията на миграция в двумерна среда поради по-сложното математическо описание на този процес. Различните опитни постановки и идентификационни методи за обработка на данните са подробно описани в специализираната литература (Fried, 1975; Bochever et al., 1979; Galabov and Pentchev, 1986; Mironenko and Rumynin, 1986; Sauty and Kinzelbach, 1988; Stoyanov, 2003).

Методите за обработка се базират на аналитични, полуаналитични или числени решения на основното уравнение на миграцията, получени при съответни начални и гранични условия за различните опитни схеми. Според използваната идентификационна процедура има два методични подхода (Stoyanov, 2003). Първият е графоаналитичен, сравнително лесно приложим, но с по-ниска точност. Параметрите се определят по характерни точки от апросимиращата опитните данни крива (Fried, 1975; Galabov, 1983; Mironenko and Rumynin, 1986; Huang, 1991). Вторият подход е по-сложен, но много по-прецизен. При него данните от опита се сравняват със серия от теоретичните криви. Сравнението се извършва ръчно с еталонни криви или автоматично посредством различни математически алгоритми (Galabov and Pentchev, 1986; Lukner and Shestakov, 1986; Sauty, 1978, 1987; Sauty and Kinzelbach, 1988; Sauty et al., 1992). Повечето от тези методи губят своята приложимост, особено след интензивното развитие на компютърните технологии. 
В момента в световната практика се налагат обновени и усъвършенствани схеми и техники за провеждане на трасерните опити, като заедно с това се разработват нови идентификационни методи и софтуерни, базирани на автоматизирано сравняване на теоретичните криви с опитните данни или с прилагане на статистически методи (Toride et al., 1995; Field, 2002, 2003; Ptak et al., 2004; Maliva, 2016). Един от универсалните и много ефективни програмни продукти за идентификация на миграционните параметри при различни схеми е разработената по проект на Френската геоложка служба BRGM компютърна програма TRAC (Gutierrez et al., 2012, 2013, Maliva, 2016). Съвместима е с новите операционни системи и с възможности потребителят да добавя собствено аналитично решение.

В няколко по-ранни публикации от автора са предложени няколко варианта на идентификационен метод за определяне миграционните параметри по данни от лабораторни и полеви опити с непрекъснато и импулсно подаване на индикатора в условията на едномерен пренос на вещество в пореста среда (Stoyanov, 2006, 2012, 2013). Програмната реализация на метода използва една от най-добрите оптимизационни процедури - алгоритъма на Levenberg-Marquardt (Marquardt, 1961). Предлаганият по-долу вариант на този метод разширява възможностите за интерпретация на данните от полеви трасерни опити с импулсен (мигновен) или непрекъснат (перманентен) източник при произволно разположение на наблюдателния сондаж спрямо точката на постьпване на индикатора. Той може да се използва и при наличие на представителна редица от данни за изменението на концентрацията на замърсителите в различни точки на водоносния хоризонт около краткотраен или непрекъснат повърхностен или подземен източник на замърсяване на подземните води.

\section{Опитни схеми}

Предложеният идентификационен метод е разработен за полеви опити с импулсен или непрекъснат източник, които се изпълняват в условията на естествен (ненарушен) подземен поток или по схемата водочерпателен опит с постоянен дебит.

Опитите в естествен поток са подходящи да се прилагат при плоско-паралелна стабилизирана структура на филтрационното поле и сравнително висока скорост на подземните води. Опитният участък включва един пусков сондаж и един или повече наблюдателни сондажа, ситуирани по посока на естествения подземен поток и под произволен ьгъл спрямо токовите линии (фиг. 1а). Фигурата илюстрира опитен участьк с един пускателен (Сп) и два наблюдателни сондажа ( $\mathrm{CH} 1$ и $\mathrm{CH} 2)$.

Като модификация на тази полевите опити в естествен поток с известна условност може се разглежда всеки източник на замърсяване на подземните води и разположените около него и по посока на естествения подземен поток мониторингови сондажи. Например като такива се приемат: (i) повърхностните аварийни разливи в ограничена площ на нефтопродукти, химически разтвори, производствени води, канализационни води и др.; (ii) хвостохранилища, депа за отпадъци, складови бази, производствени площадки, попивни ями и др.; (iii) подземно изтичане през нарушения в обсадните тръби на дълбоки проучвателни или експлоатационни сондажи на нефт, силно минерализирани води, реагентни или продуктивни разтвори и др.

Водочерпателните опити с постоянен дебит протичат в условията на плоскорадиална стабилизирана филтрация. Използват се при по-сложна структура на естествения подземен поток и/или при ниски скорости на подземните води. При равни други условия тази схема съкращава значително времетраенето на трасерния опит, поради което е и често използвана в хидрогеоложката практика. 
Опитният участък включва три сондажа - пускателен (Сп), наблюдателен (Сн) и водочерпателен (Св). Наблюдателният сондаж е разположен в участька между пускателния и черпателния сондаж и встрани от свързващата ги токова линия (фиг. 1b).

Методът за обработка на експерименталните данни е разработен при следните допускания относно условията за провеждане на полевите опити:

- миграцията протича в хомогенна и изотропна двумерна пореста среда;

- структурата на филтрационното поле е едномерна, като в зависимост от опитната схема е плоско-паралелна или плоско-радиална;

- скоростта на филтрация v е постоянна;

- фоновата концентрация на индикатора $c_{B}$ е постоянна;

- при импулсен източник индикаторът постьпва пакетно, като за много кратък период определено количество от него с маса $\Delta M$ се инжектира в пусковия сондаж;

- при непрекъснат източник общото количество индикатор с маса $M$ по време на опита постьпва в пусковия сондаж с постоянна скорост $d M / d t$;

- концентрационното поле е нестабилизирано и двумерно, като концентрацията на индикатора във водоносния хоризонт $c$ се описва с функцията $c=f(x, y, t)$;

- по време на опита в наблюдателния сондаж се следи изменението на концентрацията на индикатора $c(t)$.

ОПИТ В ЕСТЕСТВЕН ПОДЗЕМЕН ПОТОК

ПЛАН
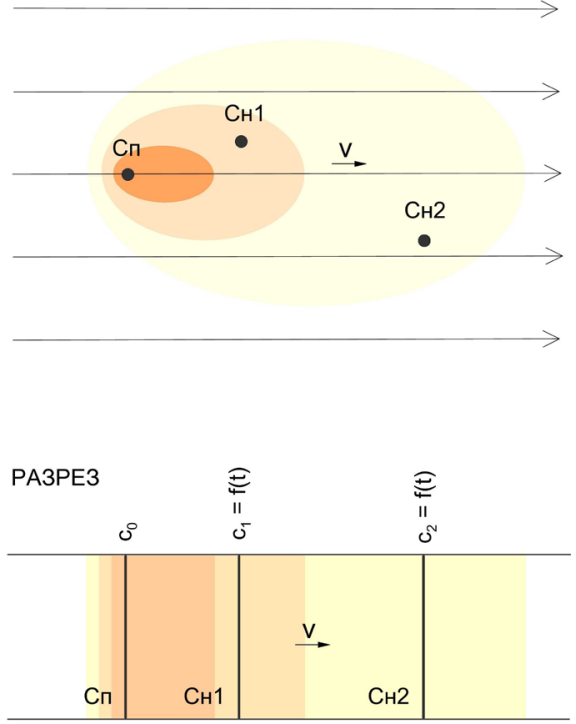

( a )
ОПИТ ПРИ ВОДОЧЕРПЕНЕ С ПОСТОЯНЕН ДЕБИТ
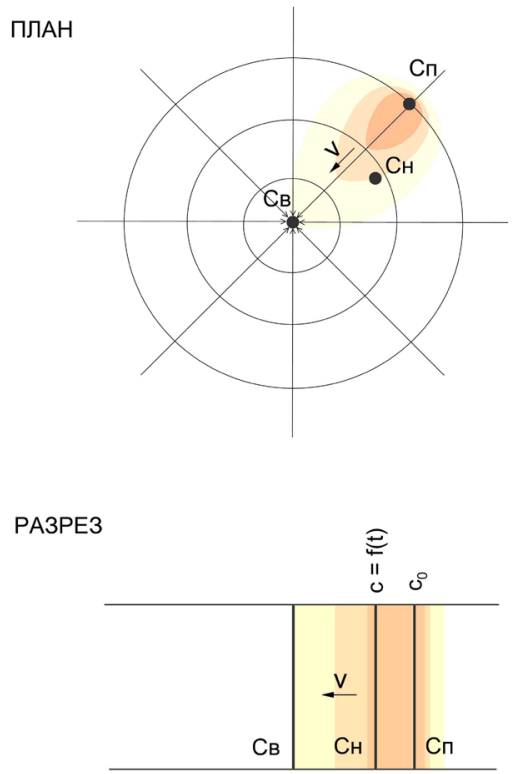

(b)

Фиг. 1. Опитни схеми за провеждане на полеви трасерни опити с импулсен или непрекъснат източник при произволно положение на наблюдателните кладенци (пример за развитието на миграционния процес при непрекъснат източник).

Fig. 1. Experimental schemes for conducting field tracer tests with a pulse or a continuous source and an arbitrary position of the observation well in respect to the tracer entry point (example revealing the mass transport process in case of a continuous source). 


\section{Математическо описание на миграцията в двумерна среда при импулсен и непрекъснат източник}

Миграцията на индикатора в условията на стабилизирана филтрация в хомогенна и изотропна двумерна пореста среда, съгласно (Bear, 1979; Kinzelbach, 1986) и с малки преобразования по отношение на константите се описва посредством частното диференциално уравнение:

$$
\frac{\partial c}{\partial t}=\frac{\alpha_{L}}{n_{S}} \frac{\partial^{2} c}{\partial x^{2}}+\frac{\alpha_{T}}{n_{S}} \frac{\partial^{2} c}{\partial y^{2}}-\frac{v}{n_{S}} \frac{\partial c}{\partial x}-\gamma c
$$

където: $c$ - концентрация на индикатора в подземните води в точка с координати $x$ и $y$ в момент $\mathrm{t}, \mathrm{ML}^{-3} ; v$ - скорост на филтрация (по Darcy), $\mathrm{LT}^{-1} ; n_{0}$ - активна порестост, без дименсия; $n_{S}-$ сорбционна порестост, без дименсия; $\alpha_{\mathrm{L}}-$ надлъжна дисперсивност, L; $\alpha_{T}$ - напречна дисперсивност, L; $\gamma$-коефициент на необратимо елиминиране, $\mathrm{T}^{-1}$.

Съществуват голям брой изведени при различни начални и гранични условия аналитични решения на уравнение (1). По-долу са представени две известни решения за импулсен и непрекъснат източник.

За импулсен източник представеното в (Hunt, 1978; Bear, 1979) решение може да се преобразува в следния вид:

$$
c(x, y, t)=\frac{\Delta M}{4 \pi m v t \sqrt{\alpha_{L} \alpha_{T}}} \exp \left(-\frac{\left(x-\frac{t v}{n_{S}}\right)^{2}}{\frac{4 \alpha_{L} t v}{n_{S}}}-\frac{y^{2}}{\frac{4 \alpha_{T} t v}{n_{S}}}\right) \exp (-\gamma t)
$$

където: $c$ - концентрация на индикатора в наблюдателния сондаж с координати $x$ и $y$ в момент $t$ след началото на опита, $\mathrm{ML}^{-3} ; \Delta M$ - масата на инжектирания в пусковия сондаж индикатор, $M$; $m$ - дебелина на водоносния хоризонт. Другите означения са същите като в уравнение (1).

За непрекъснат източник е възможно да се използва даденото в (Fried, 1975) решение след като се запише във вида:

$$
c(x, y, t)=\frac{d M / d t}{4 \pi m n_{S} v \sqrt{\alpha_{L} \alpha_{T}}} \exp \left(\frac{x}{2 \alpha_{L}}\right) W\left(\frac{r^{2} n_{S}}{4 \alpha_{L} v t}, \frac{r \gamma^{*}}{2 \alpha_{L}}\right)
$$

където: $\mathrm{dM} / \mathrm{dt}$ - количество от индикатора, което постьпва от пусковия сондаж във водоносния хоризонт за единица време, $\mathrm{ML}^{-1}$; W( ) - функция на Hantush, безразмерна. Параметрите $\gamma^{*}$ и $\mathrm{r}^{2}$ се определят по формули (4) и (5). Останалите означения са като в (1) и (2).

$$
\begin{aligned}
& \gamma^{*}=\sqrt{1+\frac{4 \gamma \alpha_{L} n_{S}}{v}} \\
& r^{2}=x^{2}+\frac{\alpha_{L}}{\alpha_{T}} y^{2}
\end{aligned}
$$

Често уравнение (3) се записва спрямо началната концентрация на

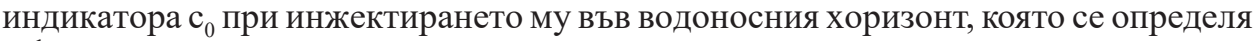
с формулата:

$$
c_{0}=\frac{d M / d t}{m n_{S} v}
$$


Предложеният метод за обработка се базира на автоматизираното сравняване на опитните данни със серия от теоретични криви, всяка от които при импулсен източник се описва с уравнение (2), а за непрекъснат източник - с уравнение (3). Множеството теоретични криви се получават чрез вариране на търсените параметри $n_{0}, n_{S}, \alpha_{L}, \alpha_{T}$ и $\gamma$, които присъстват като константи в двете уравнения. Сравнението на опитните данни с теоретичните криви се прави с оптимизационна процедура, включваща алгоритъма на Levenberg-Marquardt (Marquardt, 1961; Brown and Dennis, 1972; Stoyanov, 2003).

\section{Реализиране на метода в компютьрни програми TT2DMI и TT2DPI}

Методът е реализиран в програмният код на компютьрните програми TT2DMI и TT2DPI, която е разработена на езика C++ за операционна система Windows. Всяка програма включва четири основни модула.

Модул за въвеждане на входните данни. Модулът позволява бързо и лесно въвеждане, редактиране и подготовка на всички числени данни. Данните, които е необходимо да бъдат зададени в този модул са: координати на наблюдателния сондаж спрямо пусковия сондаж $x$ и $y, \mathrm{~m}$; скорост на филтрация $v, \mathrm{~m} / \mathrm{d}$; фонова концентрация $c_{B}, \mathrm{~g} / \mathrm{L}$; текстов файл с данни за концентрацията $c_{i}=f\left(t_{i}\right), \mathrm{g} / \mathrm{L}$, където $t_{i}$ е времето от началото на опита до $i$-тото измерване, min. Входната информация за $x, y, v$ и $c_{B}$ се въвежда в диалогов режим. Измерените в наблюдателния кладенец стойности на концентрацията $c_{i}$ в моменти $t_{i}$ се въвежда от предварително създаден външен файл с разширение *.xls. Допълнително като входни данни се задават начални стойности на търсените параметри $n_{0}, n_{S}, \alpha_{L}, \alpha_{T}$ и $\gamma$, приведената маса на индикатора $M_{a}$ и възможните граници на тяхното изменение. При обработването на данни от опити с индикатори, които не се разпадат или утаяват, идентификацията се изпълнява след изключване на параметъра $\gamma$. Приведената маса на инжектирания по време на опитите индикатор за импулсен източник се определя с израза $M_{a}=\Delta M / 4 \pi m, \mathrm{~g} / \mathrm{m}$, а за непрекъснат източник $-M_{a}=c_{0} / 4 \pi, \mathrm{g} / \mathrm{L}$.

Модул за минимизачия. Търси минимума в разликите между опитните данни и теоретичните криви при различни стойности на миграционните параметри и приведената маса като използва алгоритьма на Levenberg-Marquardt. Визуално се следи степента на несъответствието между измерените стойности и теоретичните криви. Установените разлики се редуцират чрез вариране на границите на изменение на тьрсените параметри.

Модул за моделиране на миграцията. Изчислява теоретичните стойности на концентрациите в наблюдателния кладенец $c_{i}$ по предварително зададени стойности на търсените параметри. Изчисленията се правят посредством уравнения (2) и (3).

Модул за извеждане на крайните резултати. Прави запис на крайните резултати в два външни текстови файла. Първият изходен файл съдържа стойностите на приведената маса $M_{a}$ и тьрсените параметри $n_{0}, n_{S}, \alpha_{L}, \alpha_{T}$ и $\gamma$, а във втория са записани данните от измерванията и тези от апроксимиращата ги теоретична крива. Резултатите могат да бъдат визуализирани посредством стандартните програми за графична обработка на 2D данни.

Необходимо е да се напомни, че когато се интерпретират данни от опити с инертни замърсители се определя активната порестост на средата $n_{0}$, а по данни от опити със сорбируеми замърсители се определя сорбционната порестост на средата $n_{S}$ спрямо съответния замърсител.

\section{Тестове за устойчивост на програмните решения}

Двете програми са тествани относно (i) грешката при идентификация на параметрите от теоретични криви при предварително задаване на техните стойности; (ii) шумоустойчивостта на решението. 
Първата проверка е направена като по предварително зададени стойности на $n_{S}, \alpha_{L}, \alpha_{T}$ и $\gamma$ са изчислени стойностите на концентрацията на индикатора в наблюдателния кладенец $c_{i}$ в определени моменти $t_{i}$. При изчисленията с двете програми се приема, че координатите на наблюдателния сондаж спрямо пусковия са $x=10 \mathrm{~m}$ и $y=1 \mathrm{~m}$, скоростта на филтрация е $v=1 \mathrm{~m} / \mathrm{d}$, фоновата концентрация е $c_{B}=0,3 \mathrm{~g} / \mathrm{L}$. За импулсен източник се приема, че масата на постьпилия индикатор е $\Delta M=100 \mathrm{~g}$, а дебелината на водоносния хоризонт е $m=$ $10 \mathrm{~m}$. За непрекъснат източник се приема, че концентрацията на постъпилия от пусковия сондаж замърсител е $c_{0}=10 \mathrm{~g} / \mathrm{L}$. Получените по този начин масиви с данни за изменението на концентрацията в наблюдателния кладенец $c_{i}=f(t)$ при импулсен и непрекъснат източник са обработени с програми TT2DMI и TT2DPI. Изчислените стойности на концентрациите в наблюдателния кладенец $c_{i}$ в моменти $t_{i}$ са представени с точки на фиг. 2. На същата фигура с плътна линия са дадена и получените с програмите идентификационни криви. Предварително зададените стойности на $n_{S}, \alpha_{L}, \alpha_{T}$ и $\gamma$ и резултатите от извършената с програмата идентификационна процедура са представени в табл. 1.

Таблица 1. Резултати от теста за идентификационната грешка при определяне на миграционните параметри с компютьрна програма TT2DMI

Table 1. Results of the test for the identification error in determining the migration parameters with the computer program TT2DMI

\begin{tabular}{|c|c|c|c|c|}
\hline Параметър & Дименсия & $\begin{array}{c}\text { Предварително } \\
\text { зададена } \\
\text { стойност }\end{array}$ & $\begin{array}{c}\text { Изчислена с } \\
\text { програма ТT2DMI } \\
\text { стойност }\end{array}$ & $\begin{array}{c}\text { Идентификационна } \\
\text { грешка, \% }\end{array}$ \\
\hline $\mathrm{n}_{\mathrm{S}}$ & - & 0,10 & 0,1002 & 0,2 \\
\hline$\alpha_{\mathrm{L}}$ & $\mathrm{m}$ & 0,25 & 0,2487 & 0,5 \\
\hline$\alpha_{\mathrm{T}}$ & $\mathrm{m}$ & 0,05 & 0,0513 & 2,6 \\
\hline$\gamma$ & $\mathrm{d}^{-1}$ & 0,10 & 0,0956 & 4,4 \\
\hline $\mathrm{M}=\Delta \mathrm{M} / 4 \pi \mathrm{m}$ & $\mathrm{g} / \mathrm{m}$ & 0,7958 & 0,7714 & 3,1 \\
\hline
\end{tabular}

Таблица 2. Резултати от теста за идентификационната грешка при определяне на миграционните параметри с компютърна програма TT2DPI

Table 2. Results of the test for the identification error in determining the migration parameters with the computer program TT2DPI

\begin{tabular}{|c|c|c|c|c|}
\hline Параметър & Дименсия & $\begin{array}{c}\text { Предварително } \\
\text { зададена } \\
\text { стойност }\end{array}$ & $\begin{array}{c}\text { Изчислена с } \\
\text { програма ТT2DPI } \\
\text { стойност }\end{array}$ & $\begin{array}{c}\text { Идентификационна } \\
\text { грешка, \% }\end{array}$ \\
\hline $\mathrm{n}_{\mathrm{S}}$ & - & 0,10 & 0,1003 & 0,3 \\
\hline$\alpha_{\mathrm{L}}$ & $\mathrm{m}$ & 0,25 & 0,2478 & 0,9 \\
\hline$\alpha_{\mathrm{T}}$ & $\mathrm{m}$ & 0,05 & 0,0527 & 5,4 \\
\hline$\gamma$ & $\mathrm{d}^{-1}$ & 0,10 & 0,0937 & 6,3 \\
\hline $\mathrm{M}=\mathrm{c}_{0} / 4 \pi$ & $\mathrm{g} / \mathrm{L}$ & 0,8963 & 0,8901 & 6,9 \\
\hline
\end{tabular}

Резултатите от първата проверка показват, че идентифицираните с двете програми стойности за $n_{S}$ и $\alpha_{L}$ почти съвпадат с предварително приетите за тях стойности, с вариации под $1 \%$. При определянето $\alpha_{T}$ и $\gamma$ разликите са малко по-големи - в диапазона между 2,6 и 6,3%. Това доказва достоверността 
на решението до степен близка на точността на използваното в съответната програма аналитично решение.

Втората проверка дава оценка за шумоустойчивостта на полученото решение. Тя е направена като допълнително към теоретично определените стойности на концентрацията на индикатора в наблюдателния сондаж $c_{i}$ e добавен случаен, нормално разпределен шум с отклонения от тези стойности c $1,2.5,5,10,15,20,25,30,35,40,45$ и 50 \%. Добавянето на шума цели да моделира възможни грешки от различно естество, които могат да се получат при провеждането на полевите опити. По този начин теоретичните стойности в тестовите примери ще се доближат максимално до реално измерваните в полеви условия концентрации. След зашумяването всяка от получените зависимости $c_{i}=f\left(t_{i}\right)$, е обработена с компютьрните програми TT2DMI и TT2DPI. На фиг. 3 , фиг. 4 и фиг. 5 са представени с пльтна линия идентификационните криви, получени при зашумяване на теоретичните стойности с $10 \%, 20 \%$ и 30\%. На същата фигура зашумените стойности на функцията $c_{i}=f\left(t_{i}\right)$ са дадени с точки.

Анализът на резултатите от направеното изследване за шумоустойчивост дава основание да се направят следните обобщения и изводи:

- Стойностите на сорбционната порестост $n_{S}$ (респ. на активната порестост $\left.n_{0}\right)$ се идентифицират изключително точно. Получените решения на практика не се влияят от шума във входните данни. Идентификационната грешка не надхвърля 2,5\% дори при стойност на шума 50\% (фиг. 6).

- Малко по-неустойчиво е решението за надлъжната дисперсивност $\alpha_{L}$. При стойности на шума от 10\% грешката е по-малка от 4\%, а при шум от 20 до $50 \%$ грешката достига до 14-18\%, без да надхвърля тези стойности (фиг. 7).

- Доста по-голяма е грешката при определяне на напречната дисперсивност $\alpha_{T}$. При шум до $10 \%$ тя е в интервала $2-8 \%$, но след това с покачване на шума от 10 до 50\% грешката бързо расте до стойности от 20 до 50\% (фиг. 8).

- Най-чувствителен е коефициентът на елиминиране $\gamma$. При шум до 7-8 \% грешката е в рамките до 10\%, но при шум 10-20\% рязко нараства до стойности 30-50 \%, като при шум до 50 \% достига и до 60-70 \% (фиг. 9).

$\mathrm{C}$ разработените компютьрни програми достатьчно точно могат да се интерпретират резултатите от трасерни тестове с импулсен и непрекъснат източник, ако грешките при измерването на концентрациите при провеждане на опитите не надхвърлят 10\%. Точността на идентификацията ще се повиши многократно, ако грешките при полевите измервания са сведени под 2 \%. Поради това тяхното използване е необходимо да се предшества от много добро
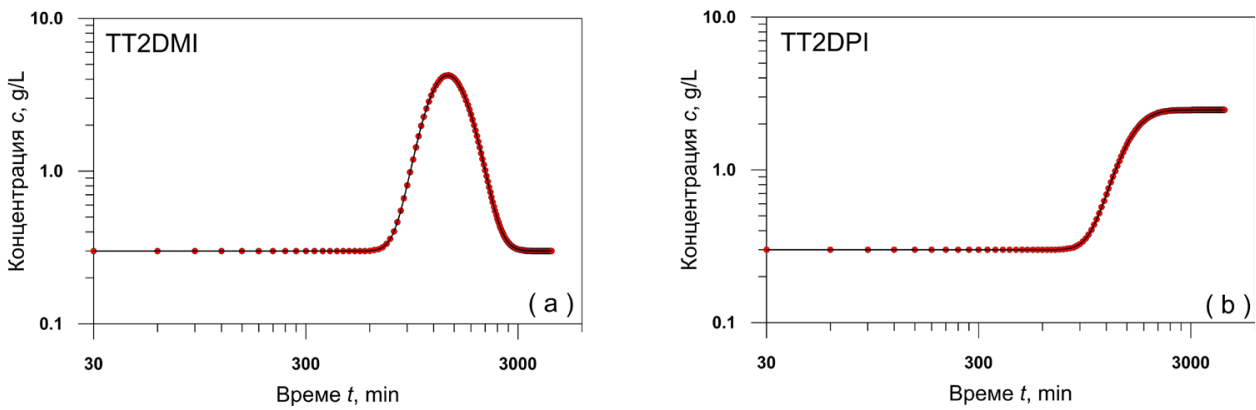

Фиг. 2. Тестови примери без зашумяване на опитните данни (a) импулсен източник, (b) непрекъснат източник.

Fig. 2. Test examples without random noise in the experimental data (a) pulse source, (b) continuous source 
планиране и изпълнение на съответната опитна схема, прецизен анализ на допуснатите по време на опитите неточности и грешки и внимателен подбор на опитните резултати.
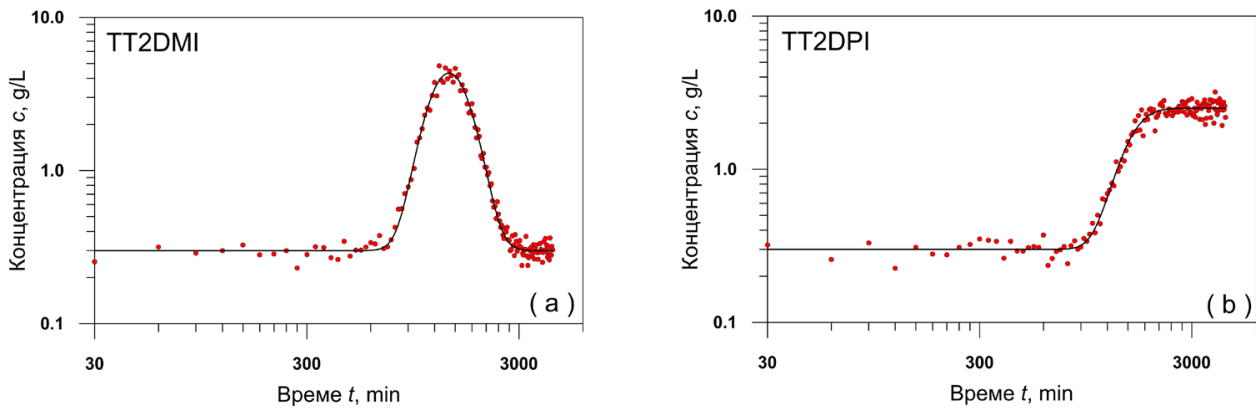

Фиг. 3. Тестови примери при зашумяване на опитните данни с 10\% (a) импулсен източник, (b) непрекъснат източник.

Fig. 3. Test examples with $10 \%$ random noise added to the experimental data (a) pulse source, (b) continuous source.
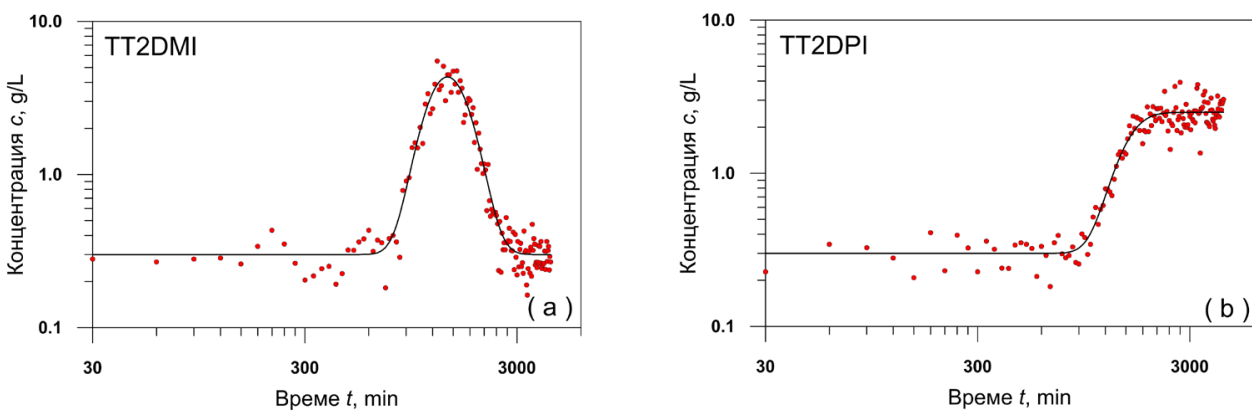

Фиг. 4. Тестови примери при зашумяване на опитните данни с $20 \%$

(a) импулсен източник, (b) непрекъснат източник

Fig. 4. Test examples with $20 \%$ random noise added to the experimental data

(a) pulse source, (b) continuous source
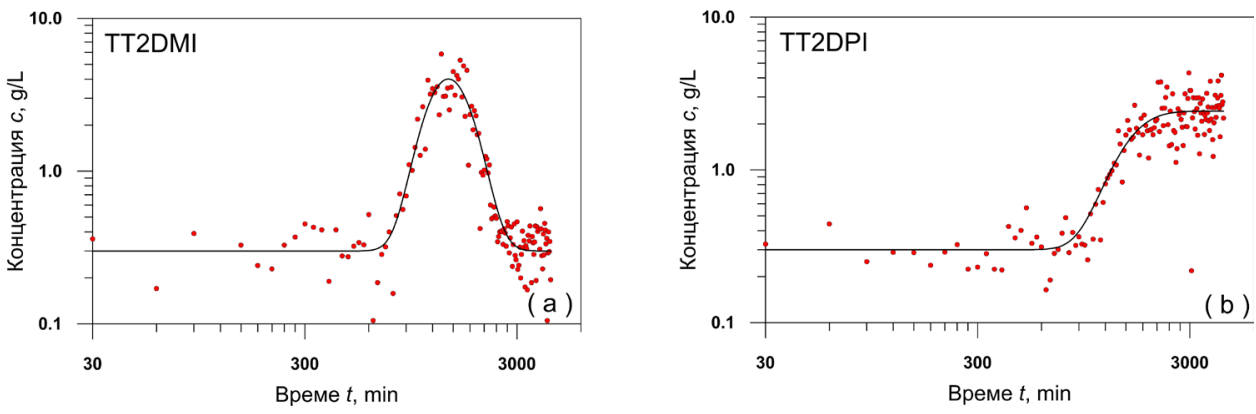

Фигура 5. Тестови примери при зашумяване на опитните данни с $30 \%$

(a) импулсен източник, (b) непрекъснат източник

Figure 5. Test examples with $30 \%$ random noise added to the experimental data

(a) pulse source, (b) continuous source 

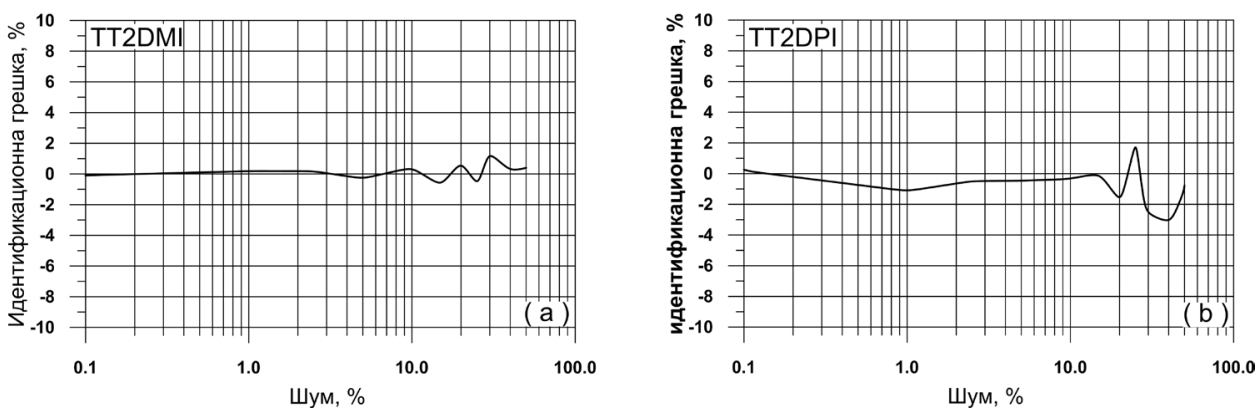

Фигура 6. Идентификационна грешка при определяне на сорбционната порестост $n_{S}$

(a) импулсен източник, (b) непрекъснат източник

Figure 6. Identification error in determining the sorption porosity $n_{S}$

(a) pulse source, (b) continuous source
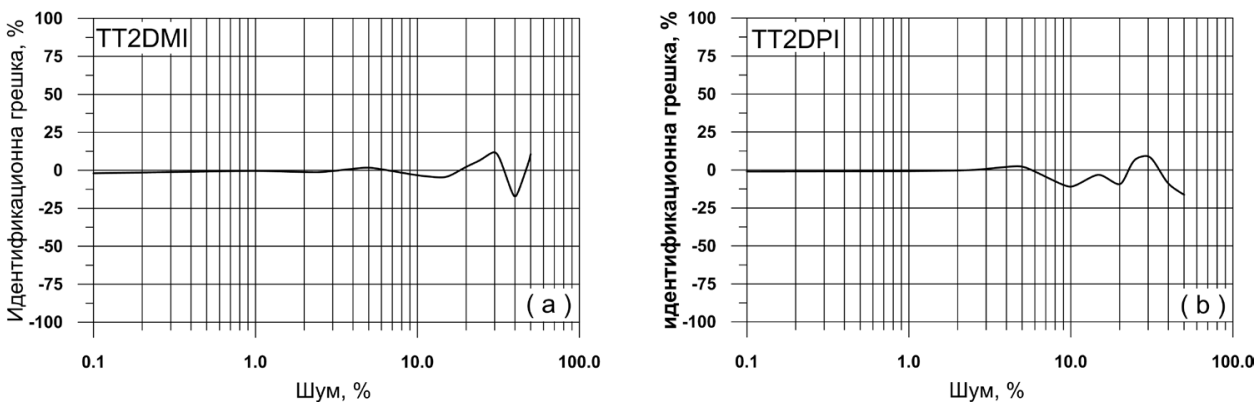

Фигура 7. Идентификационна грешка при определяне на надлъжната дисперсивност $\alpha_{L}$

(a) импулсен източник, (b) непрекъснат източник

Figure 7. Identification error in determining the longitudinal dispersivity $\alpha_{L}$

(a) pulse source, (b) continuous source
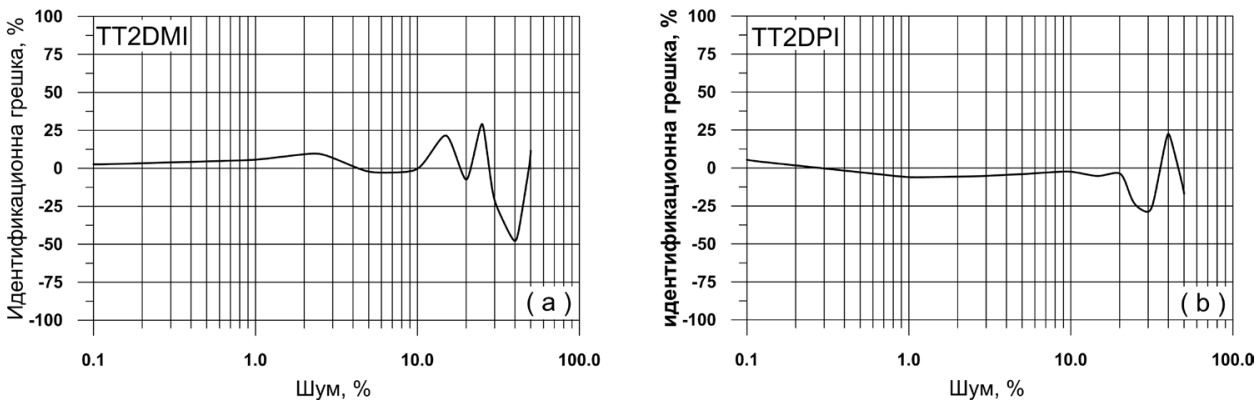

Фигура 8. Идентификационна грешка при определяне на напречната дисперсивност $\alpha_{T}$

(a) импулсен източник, (b) непрекъснат източник

Figure 8. Identification error in determining the transverse dispersivity $\alpha_{T}$

(a) pulse source, (b) continuous source 

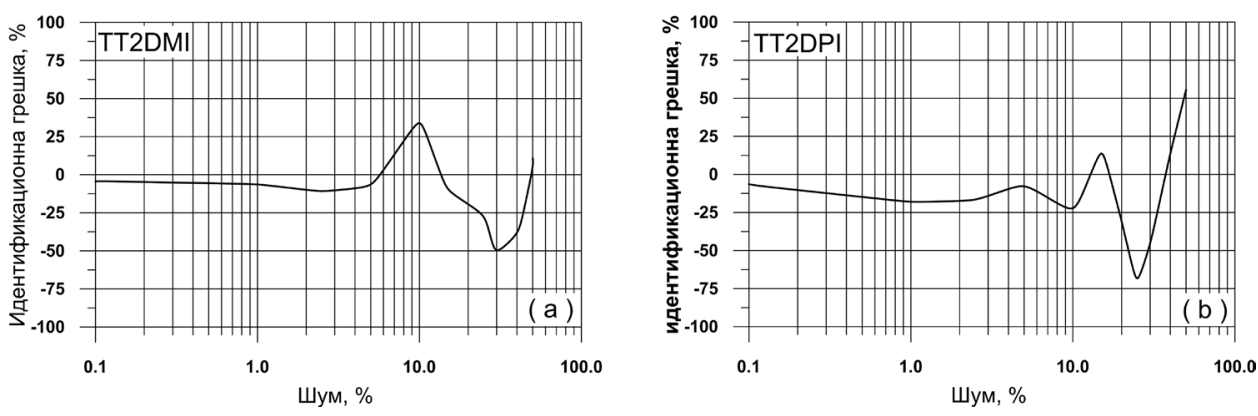

Фигура 9. Идентификационна грешка при определяне на коефициента на елиминиране $\gamma$ (a) импулсен източник, (b) непрекъснат източник

Figure 9. Identification error in determining the rate constant $\gamma$

(a) pulse source, (b) continuous source

\section{Заключение}

Направените тестове и проверки за устойчивост на решението потвърждават, че предложеният идентификационен метод и компютьрните програми TT2DMI и TT2DPI могат успешно да се използват за определяне на основните миграционни параметри $n_{0}, n_{S}, \alpha_{L}, \alpha_{T}$ и $\gamma$ по данни от полеви трасерни опити с импулсен или непрекъснат източник и произволно положение на наблюдателните сондажи.

Основни предимства на метода са бързата и напълно автоматизирана обработка на данните и високата точност на получените резултати.

\section{Литература:}

\section{References}

Bear, J. 1979. Hydraulics of groundwater. NY, McGraw - Hill. 567 p.

Bochever, F. M., N. N. Lapshin, A. E. Oradovskaya. 1979. Protection of groundwater from pollution. Publ. House Nedra, Moscow, 256 p. (in Russian).

Brown, K. M., J. E. Dennis. 1972. Derivative free analogues of the LevenbergMarquardt and Gauss algorithms for nonlinear least squares approximations. Numerishe Mathematik, 18, 289-297, https://doi.org/10.1007/BF01404679.

Field, M. S. 2002. The QTRACER2 program for tracer-breakthrough curve analysis for tracer tests in karstic aquifers and other hydrologic systems. USEPA, NCEA, Washington, DC, EPA/600/R-02/001, 175 p.

Field, M. S. 2003. Tracer-test planning using the efficient hydrologic tracer-test design (EHTD) program. USEPA, NCEA, Washington, DC, EPA/600/R-03/034, 179 p.

Freeze, R. A., J. A. Cherry. 1979. Groundwater. NY. Printice-Hall Inc. 604 p.

Fried, J. J., 1975. Groundwater Pollution. NY, Elsevier, 930 p.

Galabov, M. M. 1983. Groundwater dynamics. Publ. House Technika, Sofia, 372 p. (in Bulgarian).

Galabov, M. M., P. P. Pentchev, 1986. Solving applied hydrogeological problems by computers. Publ. House Tehnika, Sofia, 375 p. (in Bulgarian).

Gutierrez, A., T. Klinka, D. Thiéry, J. Elsass. 2012. Manuel d'utilisation de TRAC: Aide à l'interprétation de traçages en milieux poreux. Rapport RP-60660-FR, BRGM, Orleans, France, 112 p.

Gutierrez, A., T. Klinka, D. Thiéry, E. Buscarlet, S. Binet, N. Jozja, C. Défarge, B. Leclerc, C. Fécamp, Y. Ahumada, J. Elsass. 2013. TRAC, a collaborative computer tool for tracer-test interpretation. EPJ Web of Conferences, 50 (2013), TRACER 
6 - The 6th International Conference on Tracers and Tracing Methods, Art. No 03002, 8 p. https://doi.org/10.1051/epjconf/20135003002.

Huang, H. 1991. On a one-dimensional tracer model. Groundwater. 29, 1, 18-20, https://doi.org/10.1111/j.1745-6584.1991.tb00491.x.

Kinzelbach, W. 1986. Groundwater modelling - An introduction an sample programs in BASIC. Amsterdam, Elsevier, $333 \mathrm{p}$.

Lukner, L., Shestakov, 1986. Modeling of groundwater mass transport. Publ. House Nedra, Moscow, 208 p. (in Russian).

Maliva, R. G. 2016. Aquifer Characterization Techniques. Schlumberger Methods in Water Resources Evaluation Series No. 4. Springer Int. Publ. Switz., 617 p., doi. org/10.1007/978-3-319-32137-0.

Marquardt, D. W. 1961. An algorithm for least-squares estimation of nonlinear parameters. Journal Soc. Ind. Appl. Math., 2, 431-441, https://doi. org/10.1137/0111030.

Mironenko, V. A., B. G. Rumynin. 1986. Mass transport tests in aquifers. Publ. House Nedra, Moscow, 240 p. (in Russian)

Ptak, Th., M. Piepenbrink, E. Martac. 2004. Tracer tests for the investigation of heterogeneous porous media and stochastic modelling of flow and transport - a review of some recent developments. Journal of Hydrology, 294, 122-163, https:// doi.org/10.1016/j.jhydrol.2004.01.020.

Sauty, J. P. 1978. Identification des paramètres du transport hydrodispersif dans les aquifères par interprétation de traçages en écoulement cylindrique convergent ou divergent. Journal of Hydrology, vol. 39, 1-2, 69-103, https://doi.org/10.1016/00221694(78)90115-4.

Sauty, J. P. 1987. Utilisation des traceurs pour définir les périmètres de protection. Rapport BRGM 87-SGN-287-EAU. BRGM, Orleans, France, 69 p.

Sauty, J., W. Kinzelbach. 1988. On the identification of the parameters of groundwater mass transport. In: Groundwater flow and quality modelling, NATO ASI Series, 33-56, https://doi.org/10.1007/978-94-009-2889-3_2.

Sauty, J .P., W. Kinzelbach, A. Voss. 1992. Program documentation of computer aided tracer test interpretation, BRGM, Orleans, France, 64 p.

Stoyanov, N. T. 2003. Evaluation and prognostication of groundwater pollution caused by sanitary landfills. $\mathrm{PhD}$ Thesis, Sofia, University of Mining Geology St. Ivan Rilski, 215 p. (in Bulgarian).

Stoyanov, N. T. 2006. Estimation of transport parameters porous medium derived from laboratory column tests data. BULAQUA, 1, 36-41. (in Bulgarian).

Stoyanov, N. T. 2012. Estimation of transport parameters of porous medium according to data derived from tests performed with impulse input of tracer. Annual of the University of Mining Geology St. Ivan Rilski, vol. 55, Part I, 134-139. (in Bulgarian).

Stoyanov, N. T. 2013. Estimation of mass transport parameters taking into account the regional hydro-dispersion in case of an impulse input of tracer. Annual of the University of Mining Geology St. Ivan Rilski, vol. 56, Part I, 173-178.

Toride, N., F. J. Leij, M. Th. van Genuchten. 1995. The CXTFIT code for estimating transport parameters from laboratory or field tracer experiments. Version 2.0, US Salinity Laboratory, Riverside, Cal., Res. Rep. No 137, 121 p.

Постьпила: 30.11 .2021

Приета: 21.12.2021
Received: 30 November 2021 Accepted: 21 December 2021 